\title{
Experimental Assessment of Time Reversal for In-Body to In-Body UWB Communications
}

\author{
Carlos Andreu (D), ${ }^{1}$ Concepcion Garcia-Pardo ${ }^{(D)}{ }^{1}$ \\ Sergio Castelló-Palacios $\mathbb{D}^{1,2}{ }^{1,2}$ and Narcís Cardona ${ }^{1}$ \\ ${ }^{1}$ Institute of Telecommunications and Multimedia Applications (iTEAM), Universitat Politècnica de València, 46022 Valencia, Spain \\ ${ }^{2}$ Centre for Biomaterials and Tissue Engineering, Universitat Politècnica de València, 46022 Valencia, Spain \\ Correspondence should be addressed to Carlos Andreu; caranes@iteam.upv.es
}

Received 19 January 2018; Revised 30 May 2018; Accepted 18 July 2018; Published 1 August 2018

Academic Editor: Simone Morosi

Copyright (C) 2018 Carlos Andreu et al. This is an open access article distributed under the Creative Commons Attribution License, which permits unrestricted use, distribution, and reproduction in any medium, provided the original work is properly cited.

\begin{abstract}
The standard of in-body communications is limited to the use of narrowband systems. These systems are far from the high data rate connections achieved by other wireless telecommunication services today in force. The UWB frequency band has been proposed as a possible candidate for future in-body networks. However, the attenuation of body tissues at gigahertz frequencies could be a serious drawback. Experimental measurements for channel modeling are not easy to carry out, while the use of humans is practically forbidden. Sophisticated simulation tools could provide inaccurate results since they are not able to reproduce all the in-body channel conditions. Chemical solutions known as phantoms could provide a fair approximation of body tissues' behavior. In this work, the Time Reversal technique is assessed to increase the channel performance of in-body communications. For this task, a large volume of experimental measurements is performed at the low part of UWB spectrum (3.1-5.1 GHz) by using a highly accurate phantom-based measurement setup. This experimental setup emulates an in-body to in-body scenario, where all the nodes are implanted inside the body. Moreover, the in-body channel characteristics such as the path loss, the correlation in transmission and reception, and the reciprocity of the channel are assessed and discussed.
\end{abstract}

\section{Introduction}

Nowadays, Wireless Body Area Networks (WBANs) enable the interconnection between independent nodes located inside the body, on the body surface, or farther away. One of the most powerful applications of these networks is the diagnosis and medical treatment of chronic illnesses at an early stage. One example of a wireless medical device is the capsule endoscopy, which can collect pictures through the whole gastrointestinal tract, reducing the patient's discomfort [1]. Capsule endoscopy makes the early diagnosis of serious illnesses such as the Crohn's disease easier [2]. Besides, this device can facilitate for doctors the diagnosis in certain inbody areas which cannot be reached without using invasive techniques like laparoscopy. WBANs can also be used in order to monitor other implanted devices like a pacemaker [3]. Considering the multiple applications of wireless medical devices, future generation of these devices should leap forward quantitatively in terms of data rate, power consumption, and so on.

The international standard for WBANs (IEEE 802.15.6) was published and approved in 2012 [4]. In this first version, the Medical Implant Communication Service (MICS) band was established as the frequency band used when at least one of the nodes involved in the communication is implanted inside the human body. These scenarios are reflected on the standard in S1 (implant to implant), S2 (implant to body surface), and S3 (implant to external) scenarios. The main reason to choose this frequency band, which works from 402 to $405 \mathrm{MHz}$, was the robustness against the high attenuated propagation medium [5]. However, MICS band is mainly constrained in terms of data rate due to the limited available bandwidth. Although new mobile communication standards encourage the use of large bandwidths to improve current applications, the medical standard has not been changed since its first publication. This fact restrains the 
IEEE Std. 802.15.6 to allow high data rate connections, which are achieved by other telecommunication services today in force. Therefore, future revisions of the current standard for in-body communications should consider more suitable frequency ranges for this purpose. A substantial improvement in technology has always led to unthinkable new applications. For example, the use of large bandwidths could make it possible to send high-definition images, which may improve the medical diagnosis.

Many research works are focused on studying new frequency bands for in-body communications. One of the proposed frequency bands has been the Industrial, Scientific, and Medical (ISM) radio band, which covers from 2400 to $2453.5 \mathrm{MHz}$ [6]. However, this band is widely used in common telecommunication systems today in force, so a high amount of interferences among services could occur. Likewise, the available bandwidth is limited as well. For this reason, recent studies consider other possibilities to cover larger bandwidths for these applications. Concretely, a large number of these researches are focused on the frequency band from 1 to $5 \mathrm{GHz}$ [7-9]. Nevertheless, the low part of this frequency range is highly saturated with conventional technologies for mobile communications such as GSM or UMTS. Hence, the Ultra-Wideband (UWB) frequency band, which covers from 3.1 to $10.6 \mathrm{GHz}$, has emerged as a potential candidate for the revision of the current standard for inbody communications. However, some studies point out how the losses increase dramatically in the propagation medium above $5 \mathrm{GHz}$ [10]. Therefore, more research works should be devoted to study the low part of UWB and then decide whether UWB is the best candidate for this purpose.

Unfortunately, the study of the in-body channel conditions is not an easy task. For obvious moral and physical integrity reasons, laboratory tests with human subjects are practically forbidden. Hence, other ways to reproduce the electromagnetic behavior of human tissues are constantly sought. In the literature, a large number of research studies, based on measurement campaigns, which use sophisticated electromagnetic simulation software tools, can be found [7, $11,12]$. Even though measurements obtained from these tools could provide a first fair approximation, the results in real conditions could vary considerably due to the fact that a simulation software is not able to reproduce all the radio channel conditions. Moreover, the complex computations performed by using accurate anatomical models could imply the need of an expensive simulation cluster. Therefore, experts try to find other methodologies to emulate the propagation channel without using human beings. The use of living animal subjects such as pigs is an alternative used solution [13-15]. However, animal experimentation is highly restricted in a large number of countries. Furthermore, the cost of each surgery, as well as the low availability of the medical staff, make the use of this methodology with high periodicity difficult. On the other hand, the use of chemical solutions which emulate the complex relative permittivity of living tissues is widely spread $[6,16]$. These mimicking materials are known as phantoms. Performing measurements by using these phantoms can be a cost-effective solution to recreate the propagation through human tissues compared to those previously mentioned.
Phantoms with a relative accuracy within narrow frequency bands can be found in the literature $[17,18]$. Nevertheless, the reported phantoms which try to reproduce the permittivity in a large bandwidth [19-21] are far from the real values [22].

Imitating the propagation through different tissues can be an unrealizable task. Reproducing a multilayer tissue model can produce inaccurate results, since the mimicking tissue layers should be separated with plastic or similar materials. The human body tissues located in both the thoracic and abdominal regions, such as muscle, heart, skin, or small intestine, have very similar complex permittivity values [22]. Accordingly, the use of a single-tissue liquid phantom is one of the most widespread approaches $[15,16]$.

Novel healthcare applications at UWB band would depend on the performance of the radio channel. Channel diversity can encourage the use of accurate localization techniques [23] as well as diversity combining methods [24]. A lot of works study the diversity performance for on-body and off-body systems [25]. However, it is difficult to find researches which address the study of the diversity of the channel considering implanted nodes [26]. Another relevant characteristic is the reciprocity of the channel. This is clear in free space, but it should be proven when the antennas are implanted in this complex propagation medium. Assuming that the channel is reciprocal, several precoding techniques can be used in order to improve the reception [27]. In fact, it is quite relevant to apply reception improvement techniques to make up the high losses of the body tissues. A fair candidate technique could be Time Reversal. Using such technique, the energy can be focused on the intended antenna by precoding the transmitted signal in either the spatial or time domains. Accordingly, the received signal level can be increased, since a gain factor is obtained compared to sending the UWB signal without this kind of precoding. This fact can improve the radio link established among implanted sensors, compensating the high losses of the propagation medium at gigahertz frequencies [22].

The motivation of this work is based on the exhaustive analysis of the UWB band as a future candidate for communications in an unexplored propagation scenario. Since the losses in the propagation medium dramatically increase at high frequencies, the IB2IB scenario can be the intermediate step in the connection between a deeply implanted node and an external or subcutaneous one. On the one hand, the losses in the propagation channel are evaluated in order to contribute to the channel modeling in this band. On the other hand, the diversity in transmission and reception is studied to encourage the use of diversity-based methods. In addition, the Time Reversal technique is applied to achieve a power gain that increases the maximum distance between transmitter and receiver.

For this work, a measurement campaign within the low part of UWB spectrum $(3.1-5.1 \mathrm{GHz})$ is performed from an experimental point of view by using a novel IB2IB measurement setup. For that, a highly accurate phantom, which mimics the dielectric properties of the human muscle tissue, is used. The measurement setup used in this work provides a large amount of measurements with high accuracy. From the obtained results, the path loss in this scenario is computed 


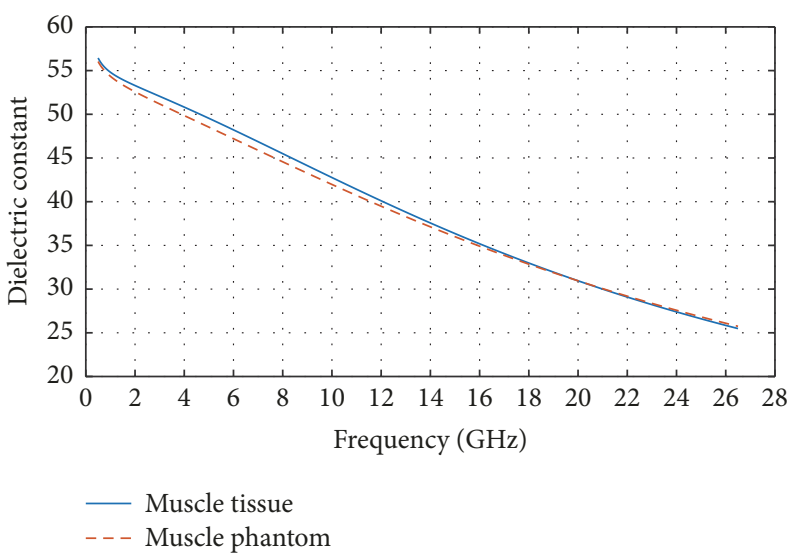

(a)

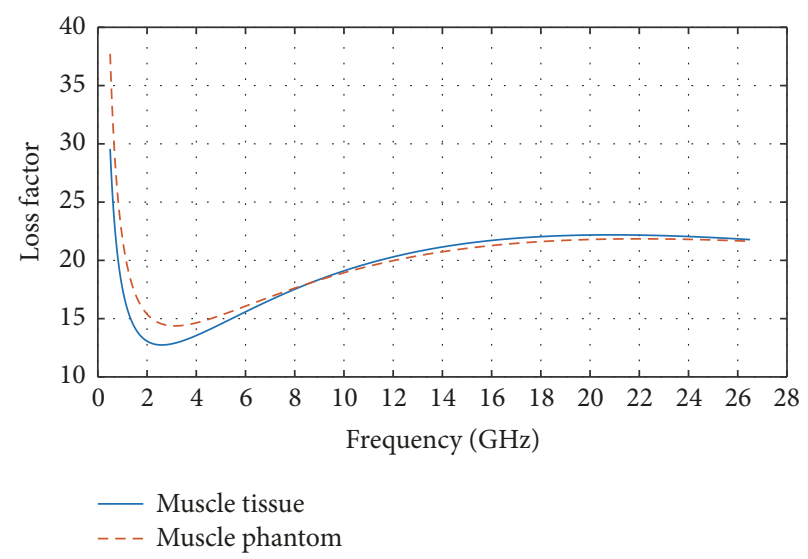

(b)

FIGURE 1: Dielectric constant (a) and loss factor (b) of the real and the muscle phantom as described in [22] and [28], respectively.

and discussed. Besides, the channel diversity in transmission and reception is evaluated for different locations of the transmitting and receiving antennas. On the other hand, the reciprocity of the propagation channel is demonstrated when both antennas are submerged into the phantom. Finally, the Time Reversal technique is analyzed to increase the received power level and, consequently, the signal-to-noise ratio (SNR).

The rest of the paper is organized as follows: Section 2 describes the measurement setup and methodology. In Section 3, the UWB in-body radio channel characteristics are shown and discussed. The Time Reversal technique is analyzed in this section as well. Finally, Section 4 summarizes the conclusions of this research study.

\section{Materials and Methods}

2.1. Experimental Scenario. According to the location of the transmitter and the receiver, different kinds of scenarios can be considered [29]. In this work, an in-body to inbody (IB2IB) scenario, where both transmitting and receiving antennas are implanted inside the body, was emulated.

2.2. High Accuracy Phantom. To reproduce the propagation medium in the abdominal region, a muscle-mimicking liquid phantom was considered. The proposed phantom is an aqueous solution of $54.98 \%$ acetonitrile and $1.07 \% \mathrm{NaCl}$ [28]. To our knowledge, the phantom used in these experimental measurements is the most accurate imitation of this tissue within the $0.5-26.5 \mathrm{GHz}$ frequency range. Figure 1 shows the comparison of both parts of the relative permittivity of the muscle phantom and their real values reported in [22].

2.3. Implanted Antennas. Typically, when antennas are intended to work in free space, designers do not care about the propagation medium, since the relative permittivity of the air is practically 1 . However, the considerations for designing implantable antenna are completely different. In this case, the wavelength is affected by the complex permittivity of the body tissues, which wrap the antenna [30]. Consequently, the antenna matching can vary considerably compared to that obtained in free space [31]. Besides, other relevant parameters such as the radiation pattern can modify its behavior as well. Therefore, human body tissues should be considered from the initial design stage, because the radiation parameters of the implanted antenna will be affected by the dielectric properties of the surrounding tissues. In the literature, a large amount of UWB antennas for biomedical applications can be found, from antennas to be tuned inside the skull [32] to tiny antennas for the capsule endoscopy [31]. In our particular case, antennas should have a compact structure to be implanted in the human torso. On the one hand, the antenna matching within the frequency band of interest should be achieved in order to perform reliable measurements. Besides, an antenna omnidirectional radiation pattern is preferred in order to communicate with a sensor network in which the nodes could be located in multiple in-body locations. Internal tissues are soft and in constant motion. Therefore, the implanted antenna can move with them and even rotate. This radiation pattern could remedy this fact. On the other hand, this kind of radiation pattern would allow interconnection with external nodes as well.

For our measurement campaign, two identical UWB monopole antenna candidates were chosen, since they comply with the requirements previously mentioned. Both antenna models are depicted in Figure 2. Hereinafter, the antenna located on the left hand side of Figure 2 will be referred to as the IB TX, whereas the other one will be the IB RX. This coplanar waveguide (CPW) monopole antenna model was designed, miniaturized, and optimized, taking into account the permittivity of the human muscle tissue by means of a direct antenna designing procedure, as described in [10]. Thus, a reflection coefficient below $-10 \mathrm{~dB}$ within our selected bandwidth of 3.1-5.1 GHz (see Figure 3), when the antennas are submerged into the phantom, was accomplished. It should be highlighted that the antennas were previously covered by a latex rubber in order to avoid their damage. This latex material is widely used in medical environments. Besides, both antennas were carefully wrapped removing the 


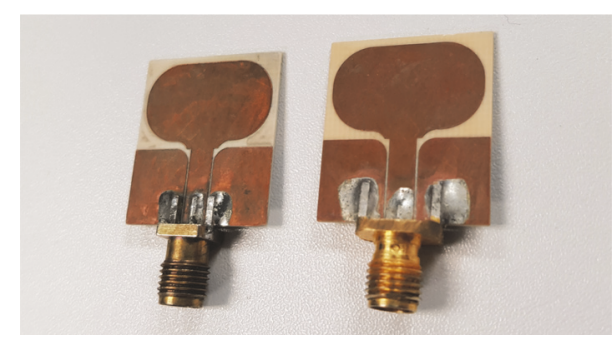

FIGURE 2: UWB in-body antennas used in the IB2IB experimental scenario.

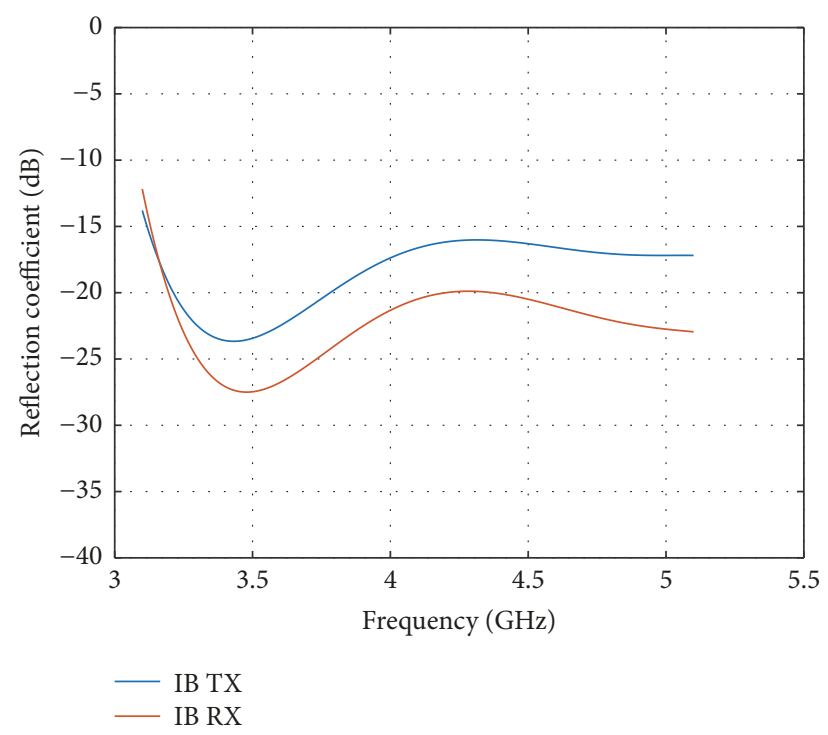

FIGURE 3: Measured reflection coefficients from 3.1 to $5.1 \mathrm{GHz}$, where both antennas were submerged into the liquid phantom.

air in order to get an airtight seal. Still, the good antenna matching remains. The values between antennas are different due to manufacturing process. Also, a quasi-omnidirectional radiation pattern, within this frequency range, considering the medium, was achieved [10]. The overall size of this antenna model was $23 \times 20 \mathrm{~mm}^{2}$. It is worth mentioning that this antenna has been used in previous measurement campaigns, using two different kinds of phantom $[10,33]$.

2.4. Methodology. The measurements were performed by a Keysight E5072A ENA vector network analyzer (hereinafter referred to as VNA). The four scattering parameters $\left(S_{11}, S_{21}\right.$, $S_{22}, S_{12}$ ) were obtained by the VNA. The transmitting and receiving antennas were connected to the ports 1 and 2 of the VNA by two coaxial cables, respectively. In order to remove the effects of cables and connectors, the VNA was calibrated through a full 2-port calibration from 3.1 to $5.1 \mathrm{GHz}$ by using a calibration kit Rosenberger 03K30R. The configuration of the VNA was as follows: output power $=8 \mathrm{dBm}$; resolution bandwidth $=3 \mathrm{kHz} ; \mathrm{N}=1601$ resolution points. In order to increase the SNR, five snapshots of every scattering parameter were obtained and averaged in each measurement point. In this scenario, the noise floor was at $-90 \mathrm{dBm}$. Moreover, the return losses of both antennas were previously measured to ensure the antenna matching during the measurements (see Figure 3).

The distance between antennas was obtained by a 3D electromagnetic tracking system. This guidance system consists of a mid-range transmitter (hereafter referred to as the tracking unit) and two magnetic sensors. The tracking unit generates a tracking volume and measures the absolute position of the sensors within this volume. The origin of coordinates is located in the center of the tracking unit. The tracking unit was close enough so that the generated tracking volume completely covered the area where the measurements were performed. From the absolute location of each sensor, the distance between them was computed. The error of the tracking system is lower than $1.4 \mathrm{~mm}$ per sensor. In order to reduce this error, 500 measurements of the sensor locations, in each measurement point, were obtained and averaged.

2.5. Experimental Measurement Setup. Figure 4 depicts the experimental IB2IB scenario used in these measurements. Firstly, the muscle-like liquid phantom was poured into a Polypropylene (PP) container. The dimensions of the phantom container $\left(30 \times 30 \times 15 \mathrm{~cm}^{3}\right)$ were chosen in order to imitate a cross section of the human abdominal region [34]. The wall thickness of the phantom was supposed to be negligible. Both antennas were submerged into the liquid phantom. Previously, the magnetic sensors were attached to the center of both antennas. The tracking unit was placed close to the phantom container, ensuring that the tracking volume covered all the sensor locations. Then, the antennas and sensors were covered by a latex rubber in order to avoid damaging the connectors. It is worth mentioning that the antenna matching is maintained despite the inclusion of this material, as can be observed in Figure 3.

The in-body transmitter (IB TX, Figure 4) was moved to multiple in-body $3 \mathrm{D}$ locations into the liquid phantom by means of a 3-axis automatic robotic arm (hereafter referred to as positioner). The positioner held and placed the antenna in different $(x, y, z)$ locations. The error of this positioner is less than $0.254 \mathrm{~mm}$ per $\mathrm{cm}$ in each axis. The in-body transmitter was moved in steps of $\Delta x=\Delta y=\Delta z=1 \mathrm{~cm}$ along the three axes, whereas the in-body receiver (IB RX) was fixed into the phantom (see Figure $4(\mathrm{a})$ ). The transmitting antenna was located in a XYZ mesh of $6 \times 7 \times 5$ measurement points. This area tries to simulate the interconnection among implanted nodes in a specific area inside the human abdomen. To facilitate the understanding of the measurements, reference points in each axis were established. The plane $z=0$ was the center plane, whereas the other planes were located above $(-\Delta z,-2 \Delta z)$ and below $(\Delta z, 2 \Delta z)$, from $\mathrm{z}=0$. The $\mathrm{Z}$ reference plane was at a height of $8 \mathrm{~cm}$ from the container's floor. $\mathrm{X}=0$ was at $15 \mathrm{~cm}$ from the container's walls, and the transmitting antenna was moved backwards from this position. Finally, $\mathrm{Y}=0$ was in the middle of the wall of the box, and the transmitter was moved to the left and right from this point. During measurements, the positioner stopped in each location, until the VNA and the tracking unit had finished the data acquisition. Software was developed with 


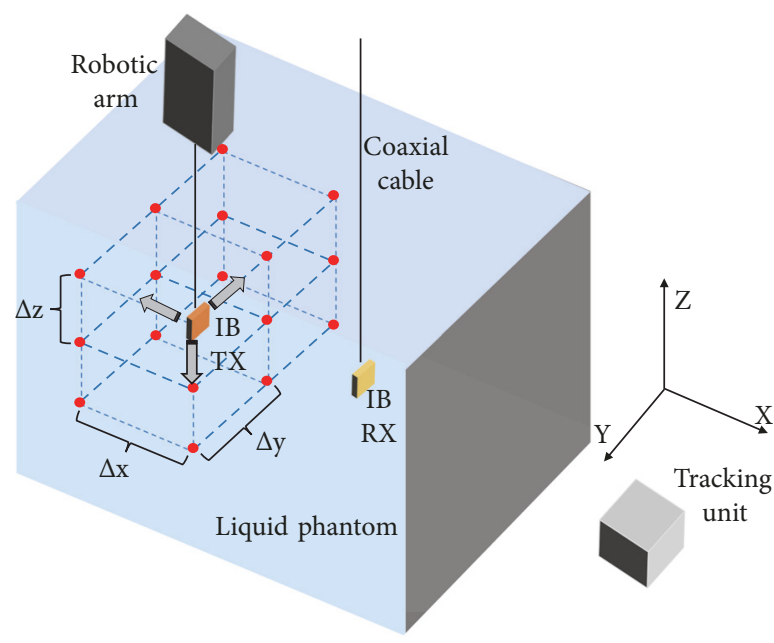

(a)

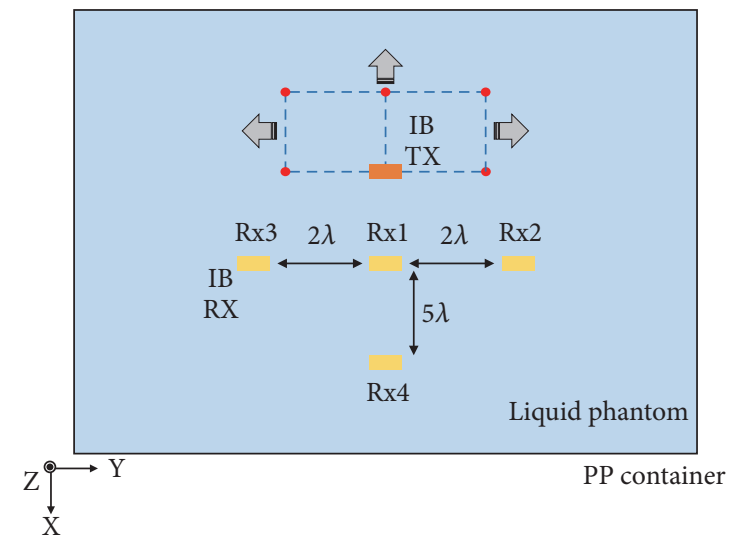

(b)

FIGURE 4: IB2IB experimental measurement setup. Locations of the transmitting (a) and receiving (b) antennas.

Visual Studio in order to control, synchronize, and measure, with the VNA, the tracking system and the positioner.

The XYZ mesh of the $6 \times 7 \times 5$ measurement points was measured in four different locations of the in-body receiver (see Figure 4(b)). These locations were named as RX1, RX2, $\mathrm{RX} 3$, and RX4. RX1 was at $\mathrm{Z}=0, \mathrm{Y}=0$ and $10 \mathrm{~cm}$ from the container's wall located behind it ( $\mathrm{X}$ axis). The rest of the receiver locations were at multiples of the wavelength $(\lambda)$ from $R X 1$ within the same height ( $Z$ plane). The value of $\lambda$ corresponds to the wavelength at the central frequency $(4.1 \mathrm{GHz})$ of the bandwidth under analysis considering the propagation speed inside the phantom.

2.6. Data Processing. From the $S_{21}$ measured samples, the path loss values from 3.1 to $5.1 \mathrm{GHz}$ for this IB2IB scenario were computed. It should be highlighted that only those $S_{21}$ values of $10 \mathrm{~dB}$ above the noise level were taken into account. Moreover, wall reflections and nondirect contributions were highly attenuated by the losses into the phantom. Thus, the received power was practically the power of the direct path contributions. In Figure 5, the power delay profile for 5 different depths in the $\mathrm{X}$ axis ranging from 5 to $9 \mathrm{~cm}$ considering $\mathrm{RX} 1$ is depicted. As can be observed, the direct path is practically the main contribution.

The path loss values were calculated as follows:

$$
P L(d B)=-10 \log _{10}\left(\overline{|H(f)|^{2}}\right)
$$

where $H(f)$ is the frequency transfer function in $N$ resolution points calculated from $S_{21}$ samples as $H(f)=\left|S_{21}\right| e^{-j \phi_{S_{21}}}$, with $\left|S_{21}\right|$ and $\phi_{S_{21}}$ being module and phase of $S_{21}$, respectively.

The diversity of the channel in transmission and reception, as well as the reciprocity of the propagation channel, were assessed by computing the correlation coefficients.

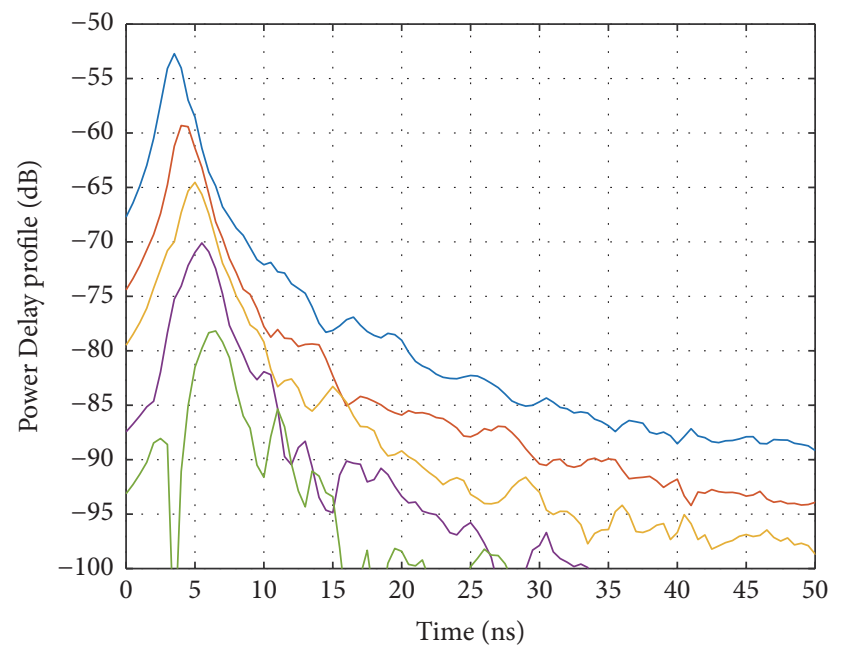

Figure 5: Power delay profile for 5 different depths in the $\mathrm{X}$ axis considering RX1.

These coefficients were calculated as the maximum correlation between two different channel impulse responses as follows:

$$
\rho_{d_{1}, d_{2}}(\tau)=\frac{E\left[h_{d_{1}}(\tau) \cdot h_{d_{2}}^{*}(\tau)\right]}{\sqrt{E\left[\left|h_{d_{1}}(\tau)\right|^{2}\right] \cdot E\left[\left|h_{d_{2}}(\tau)\right|^{2}\right]}},
$$

$E$ [] being the expected value and $h_{d_{i}}(\tau)$ being the channel impulse response for a distance between antennas $d_{\mathrm{i}}$. It is worth mentioning that the $S_{21}$ response measured by the VNA is directly the channel transfer function $(H(f))$ [35]. During the measurement time, a static channel was considered. In order to obtain the channel impulse response, a rectangular windowing function was applied so as not to lose resolution [36]. Then, the channel impulse responses 


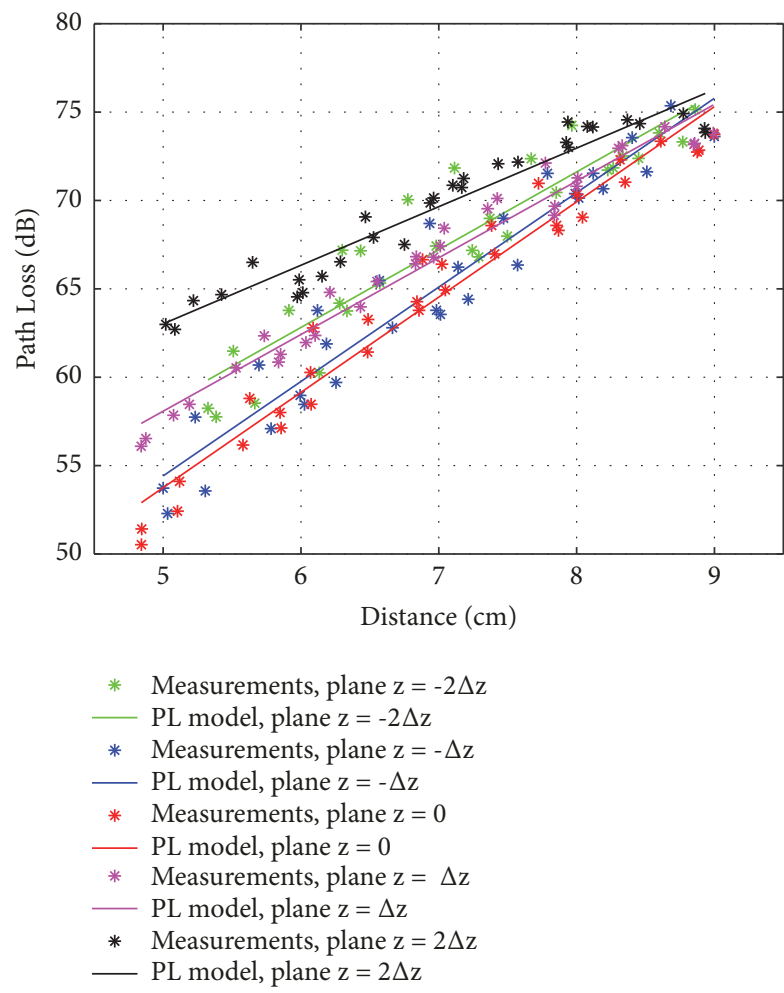

(a)

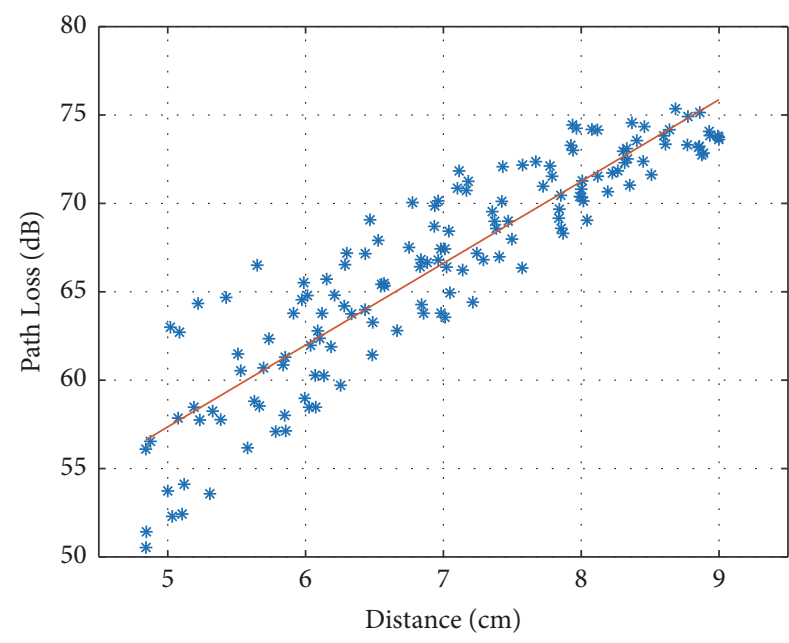

* Measurements

— PL model

FIGURE 6: Path loss as a function of distance between antennas considering RX1. Values and models for different heights (a) and all the measured points (b).

were obtained by the IFFT with $\mathrm{N}=1601$ resolution points as in [33].

\section{Results and Discussion}

\subsection{Channel Characteristics}

3.1.1. Path Loss. For this section, the in-body receiver was at RX1 (see Figure 4(b)). Figure 6 depicts, on the one hand, the path loss for different heights, i.e., $Z$ planes (see Figure 6(a)), and, on the other hand, the path loss considering all the values involved (see Figure 6(b)). As can be observed, the linear approximation model achieves a well fitting in all cases. The linear model can be expressed as follows:

$$
P L_{z}(d B)=P L_{0, z}+\alpha_{z} \cdot d(\mathrm{~cm})
$$

where $P L_{0, z}$ is the value of the path loss for either different $\mathrm{Z}$ planes or all of them when the distance between antennas $d$ tends to 0 ; and $\alpha_{z}$ is a fitting parameter.

Table 1 presents the path loss fitting parameters considering different heights as well as all the measured values. As can be observed from this table, the values are quite similar. $P L_{0, \mathrm{z}}$ varies from 34 to $46 \mathrm{~dB}$, and $\alpha_{\mathrm{z}}$ varies from 3 to 5 , approximately. It can be noted that those furthest planes from $\mathrm{z}=0(-2 \Delta \mathrm{z}, 2 \Delta \mathrm{z})$ have more losses than the rest. This is due to the fact that the antenna misalignment increases at the extreme planes. The losses are lower when the antennas are
TABLE 1: Fitting parameters of the approximation model for the IB2IB scenario.

\begin{tabular}{lcc}
\hline Z plane & $\mathrm{PL}_{0, \mathrm{z}}$ & $\alpha_{\mathrm{z}}$ \\
\hline$-2 \Delta \mathrm{z}$ & 36.4233 & 4.3987 \\
\hline$-\Delta \mathrm{z}$ & 27.7087 & 5.3416 \\
\hline 0 & 26.8048 & 5.3894 \\
\hline$\Delta \mathrm{z}$ & 36.3740 & 4.3404 \\
\hline $2 \Delta \mathrm{z}$ & 46.4496 & 3.3141 \\
\hline All measured points & 34.2116 & 4.6290 \\
\hline
\end{tabular}

at the same height $(\mathrm{z}=0)$. When all the measured points are considered, the slope of the approximation model is higher than those obtained for different heights. However, there is no a significant variation.

Our results evidence that the path loss values are well fitted by a linear model in all the cases. The path loss approximation model for the measured points at all $\mathrm{Z}$ planes is consistent with the values obtained in a previous measurement campaign in which the receiver antenna was quite large [33]. Moreover, the trend of our approximation models is in agreement with other phantom-based measurements as [6]. Nevertheless, the conclusions of comparing both studies could be counterproductive since the frequency bands assessed in each study are different. For future implanted sensor networks, many more measurements should be 


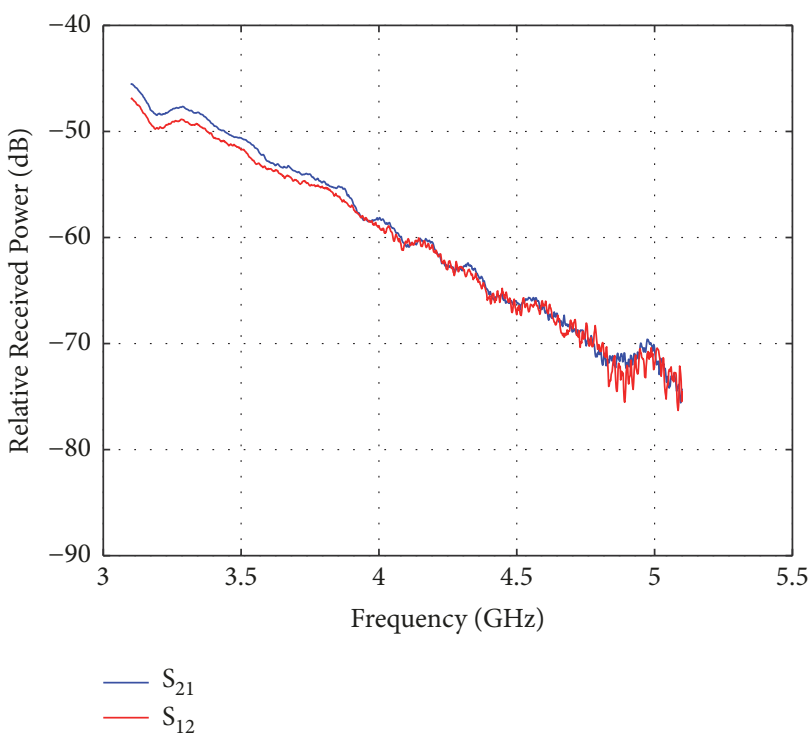

(a)

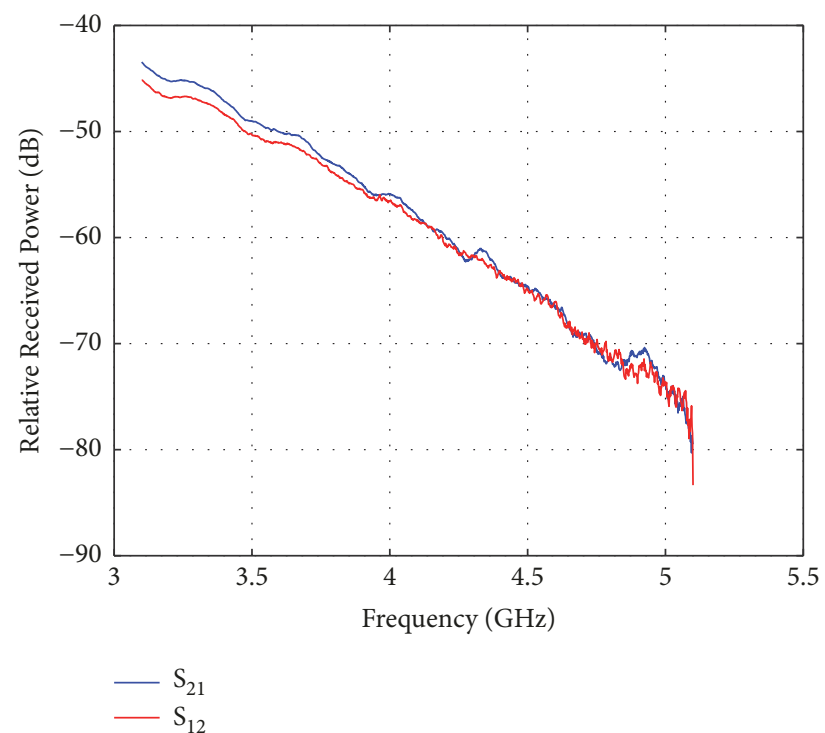

(b)

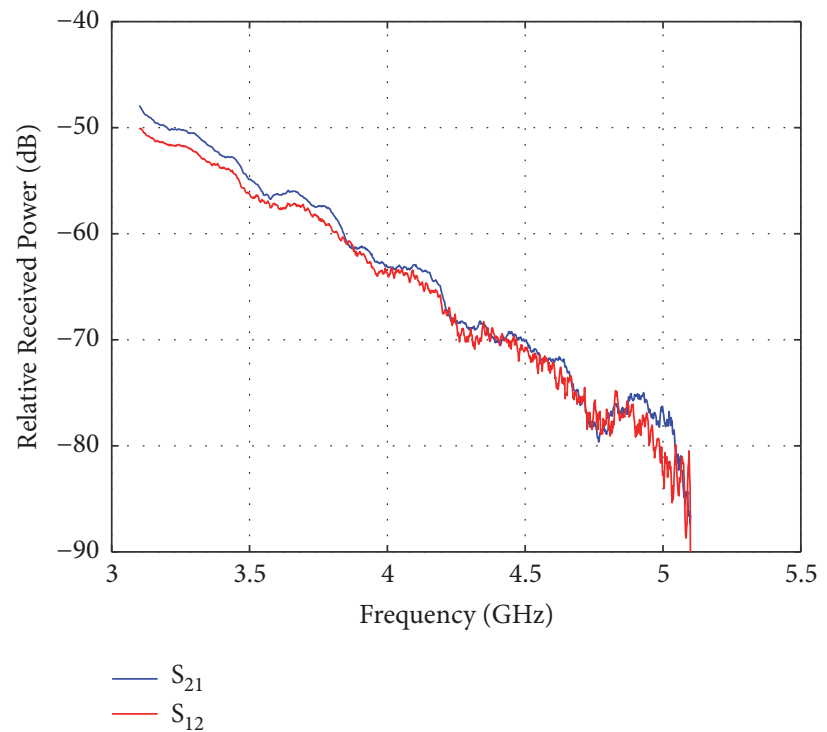

(c)

FiguRE 7: Forward transmission coefficient in both directions (from port 1 to port 2 and vice versa) in three transmitting antenna locations at different $\mathrm{Z}$ planes. $\mathrm{Z}=-\Delta \mathrm{Z}(\mathrm{a}), \mathrm{Z}=0(\mathrm{~b})$, and $\mathrm{Z}=\Delta \mathrm{Z}(\mathrm{c})$.

performed, because the related literature lacks these kinds of studies in this promising scenario.

3.1.2. Reciprocity of the Propagation Channel. A reciprocal channel means that the forward transmission coefficients $\left(S_{21}, S_{12}\right)$ have similar features in both communication directions. If the reciprocity of the channel is ensured, techniques can be used from which to benefit [37]. When both antennas are located in free space with line-of-sight (LOS) conditions, it is relatively obvious to think that the channel is reciprocal. At in-body communications, the antennas are located in the body, which produces a high variation in their characteristics. The complex permittivity of the body tissues modifies the propagation speed and therefore the wavelength compared to free space. Thus, the field regions might change their radiation areas [38]. Therefore, the antennas could be in near field regions, where the radiation parameters fluctuate considerably. On the other hand, even though a single-layer phantom is used, the LOS cannot be guaranteed, since there is a chemical solution between antennas as well as the protective layers that wrap the antennas.

Figure 7 depicts $S_{21}$ and $S_{12}$ in three different measurement points at different heights. For this study, the values obtained from RX1 are used as well. It can be noted that the frequency responses are practically identical to each other. Besides, Table 2 shows the probability values where the correlation between $S_{21}$ and $S_{12}$ in the same measurement point at different $\mathrm{Z}$ planes is above 0.8 . One can observe the 
TABLE 2: Probability that correlation between $S_{21}$ and $S_{12}$ is greater than 0.8 .

\begin{tabular}{lc}
\hline$Z$ plane & Probability $>0.8$ \\
\hline$-2 \Delta z$ & 0.8571 \\
\hline$-\Delta z$ & 0.9310 \\
\hline 0 & 0.9355 \\
\hline$\Delta z$ & 0.9032 \\
\hline $2 \Delta z$ & 0.8000 \\
\hline
\end{tabular}

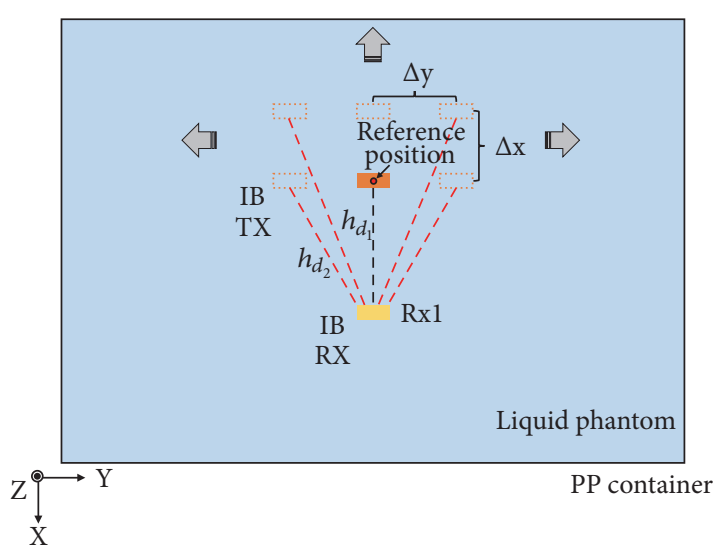

FIGURE 8: Scheme for the assessment of the correlation in transmission.

high similarity regardless of the location of the transmitting antenna. Hence, it can be concluded that the propagation channel can be practically considered reciprocal when both antennas are submerged into the liquid phantom.

\subsubsection{Correlation Coefficients}

(a) Correlation in Transmission. The diversity of the channel was evaluated by the correlation coefficients. In Figure 8, the scheme for computing the correlation coefficients in transmission is shown. The receiving antenna was in the $\mathrm{RX} 1$ position. In each $\mathrm{Z}$ plane, a reference location of the transmitting antenna was established. These reference locations were at $\mathrm{X}=0, \mathrm{Y}=0$ in each $\mathrm{Z}$ plane. Accordingly, the channel impulse response in the reference location $\left(h_{d}\right.$ in (2)) was correlated with those impulse responses located at the same $\mathrm{Z}$ plane $\left(h_{d_{2}}\right.$ in (2)).

Figure 9 depicts the correlation in transmission for the transmitting antennas located at the same height, particularized for RX1. From this figure, it can be noted that the behavior of the correlation is quite similar regardless of the $\mathrm{Z}$ plane. Besides, it can be observed that the correlation remains constant when the distance in $\mathrm{X}$ axis is fixed and the distance in $\mathrm{Y}$ axis is varied. Moreover, the correlation drops below 0.5 when the transmitter location is above $4 \Delta \mathrm{x}(4 \mathrm{~cm})$ from the reference transmitting antenna location.

(b) Correlation in Reception. In order to assess the correlation in reception, the position of different receiving antenna locations was considered. The channel impulse responses for each transmitting antenna location, obtained from RX2, were correlated with those obtained from the rest of the receivers (RX1, RX3, and RX4) at the same receiving antenna location. According to (2), $h_{d_{1}}$ was the channel impulse response obtained from RX2 in a transmitting antenna location, whereas $h_{d_{2}}$ was the impulse response in the same antenna location, obtained from the other receiver locations, i.e., $d_{1}=$ $d_{2}$. RX1 and RX3 were $2 \lambda$ and $4 \lambda$ away from RX2 in X axis, respectively, while RX4 was $2 \lambda$ away in $\mathrm{X}$ axis and $5 \lambda$ in $\mathrm{Y}$ axis from RX2 as well (see Figure 4(b)).

Figure 10 shows the complementary cumulative distribution function (CCDF) for the correlation in reception, i.e., $\mathrm{CCDF}=1-\mathrm{P}(X<$ correlation coefficients $)=\mathrm{P}(X>$ correlation coefficients). The CCDF in each pair of receivers was computed considering all the measurement points in the three axes. As can be observed, the correlation decreases as the distance between receivers increases. This is more significant when the receiving antennas are away in both axes (RX2 and RX4), since practically all the correlation coefficients are below 0.65 . When the receiver antennas are only separated in the $\mathrm{X}$ axis, the correlation increases considerably. Nevertheless, the increment of the distance between receivers in the $\mathrm{X}$ axis can reduce the correlation. As can be seen in Figure 10, the values of the correlation coefficients when the receiving antennas are separated $4 \lambda$ (RX2 and RX3) decrease by 0.1 units compared to the values when receivers are separated $2 \lambda(\mathrm{RX} 2$ and $\mathrm{RX} 1)$. However, the correlation was very high in both cases.

\subsection{Time Reversal Technique}

3.2.1. Principle. In 2004, the Time Reversal (TR) signal processing was extended to the electromagnetic waves [37]. This technique was used to focus the transmitted energy on the intended receiver, thus reducing the interference from other field sources.

Combining UWB pulses and TR can take benefit from multipath propagation environments [27]. TR achieves a space-time compression. The idea is to precode the transmitted UWB pulse to obtain a new channel impulse response. Firstly, the receiver sends a known pulse to the transmitter. Then, from such received pulse, the transmitter can estimate the channel impulse response, $h(\tau)$, between transmitter and receiver. Thus, the transmitter sends a precoded signal to the receiver, which consists of the signal to be transmitted, $x(t)$, convolved with the inverse and conjugated channel impulse response previously estimated, $h^{*}(-\tau)$. It should be remarked that for applying this technique the channel should be reciprocal so that the channel impulse response can be estimated [37].

Mathematically, the received signal $y(t)$ can be expressed as

$$
\begin{aligned}
y(\tau) & =\left(x(\tau) \otimes h^{*}(-\tau)\right) \otimes h(\tau) \\
& =x(\tau) \otimes\left(h^{*}(-\tau) \otimes h(\tau)\right)=x(\tau) \otimes h_{e q}(\tau)
\end{aligned}
$$

where $\otimes$ is the convolution operator and $h_{e q}(\tau)$ is the equivalent channel impulse response, obtained from

$$
h_{e q}(\tau)=h^{*}(-\tau) \otimes h(\tau) .
$$




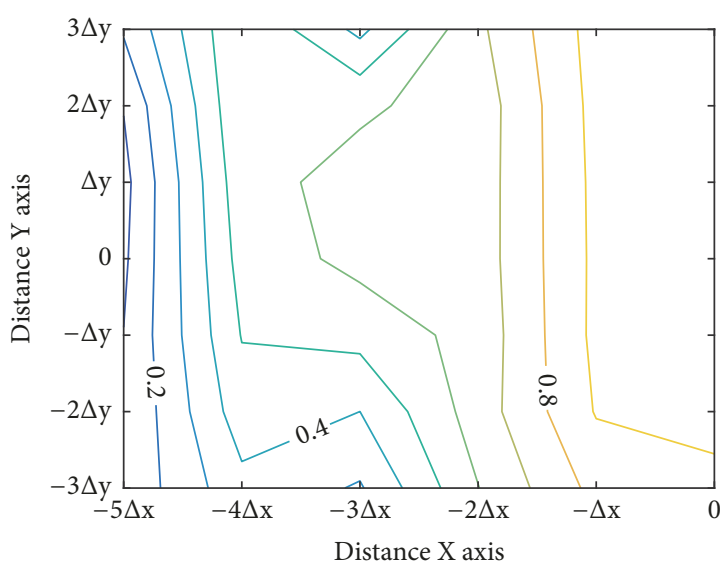

(a)

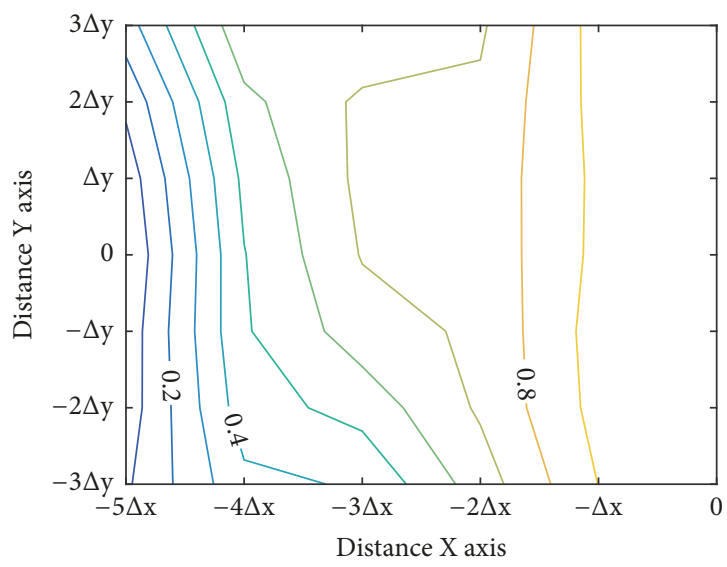

(c)

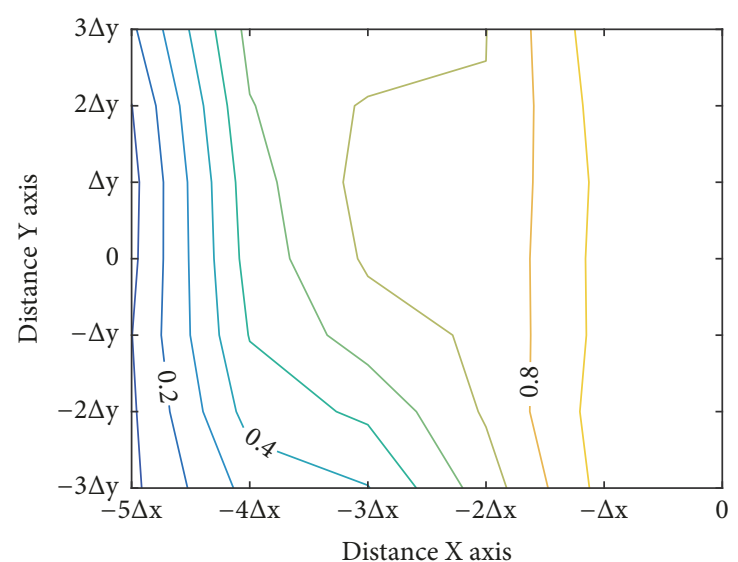

(b)

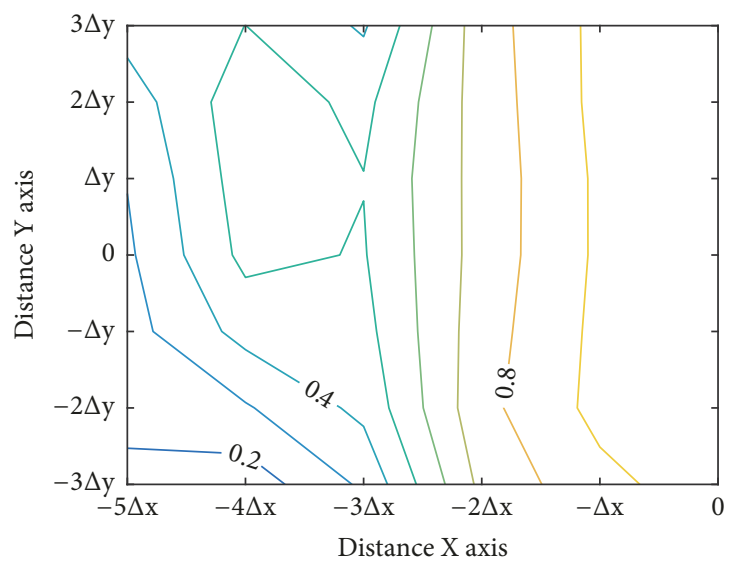

(d)

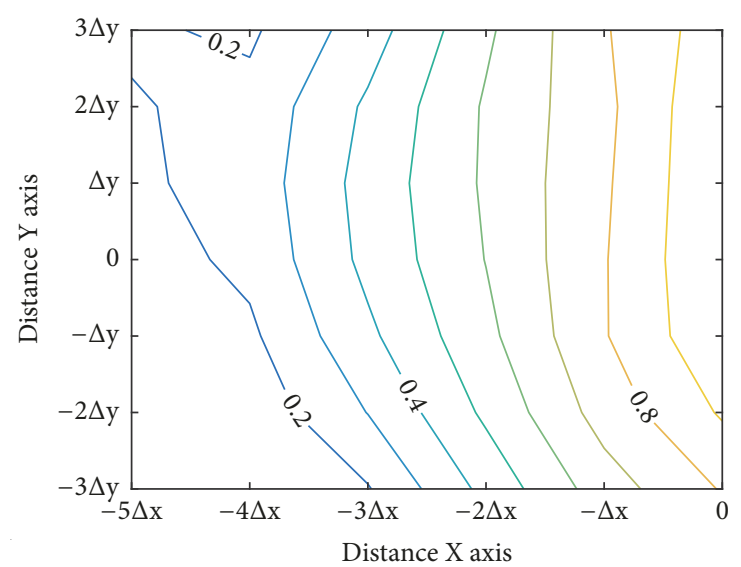

(e)

FIGURE 9: Correlation coefficients at different heights in the experimental IB2IB scenario using RX1. $Z=-2 \Delta z(a), Z=-\Delta z$ (b), $Z=0$ (c), $Z=$ $-\Delta \mathrm{z}(\mathrm{d})$, and $\mathrm{Z}=2 \Delta \mathrm{z}(\mathrm{e})$.

$h(\tau)$ and $h^{*}(-\tau)$ are the channel impulse response and its inverted and conjugated form, respectively.

To evaluate the characteristics of the TR technique, some figures of merit can be found in the literature. In [27], authors proposed a peak to peak gain $\left(G_{p 2 p}\right)$ which shows the ratio between the strongest path using TR $\left(h_{e q}\right)$ and the conventional form $(h)$. Therefore, the precoding of the channel could achieve a gain in the received signal, which can be measured as follows:

$$
G_{p 2 p}=10 \log 10\left(\frac{\max \left(\left|h_{e q}(\tau)\right|^{2}\right)}{\max \left(|h(\tau)|^{2}\right)}\right)
$$




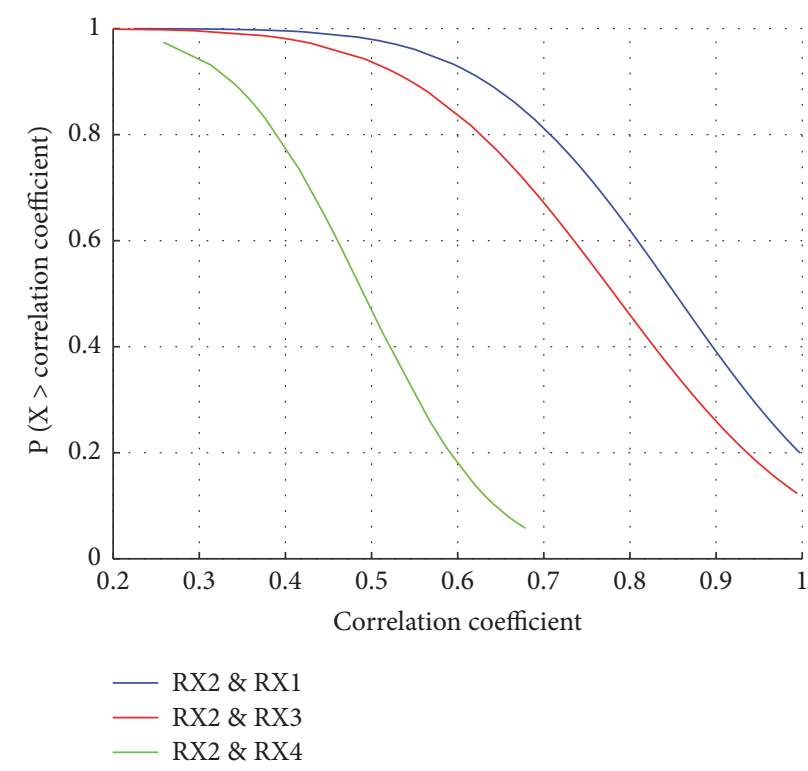

FIgURE 10: Complementary cumulative distribution function (CCDF) for the correlation coefficients in reception.

3.2.2. Analysis. As can be observed in Section 3.2, Figure 6, losses dramatically grow as the distance between antennas increases in this complex environment.

In this section, TR is used in the IB2IB scenario described in Figure 4. Regarding the receiving antenna, it was placed in the RX1 position. As mentioned in Section 2.6, the received power is practically the direct path contribution. Therefore, the best way to evaluate the performance of TR is to compare the strongest path with and without using TR. Accordingly, $G_{p 2 p}$ was calculated for each $\mathrm{Z}$ plane to evaluate the performance of TR in this specific application. As evidenced in Section 3.1.2, a reciprocal channel can be assumed. Hence, according to (4), the estimated channel impulse response $h^{*}(-\tau)$ was calculated from the $S_{12}$ samples, whereas $h(\tau)$ was the channel impulse response obtained from the $S_{21}$ samples.

Figure 11 shows the values of the peak to peak gain for each $\mathrm{Z}$ plane. The gain approximately varies from 4 to 11 $\mathrm{dB}$, being higher when transmitter and receiver are further away from each other. Besides, the gain has higher values when the transmitter is located at the upper and lower planes $(-2 \Delta z, 2 \Delta z)$. As can be observed in Figure 6, the losses are $5 \mathrm{~dB} / \mathrm{cm}$, approximately. Therefore, by using TR technique, the distance between transmitters could be increased from 1 to $2 \mathrm{~cm}$, approximately. It implies $11-22 \%$ of that without applying Time Reversal processing. On the other hand, with the observation of the diversity in transmission shown in Figure 9, it can be noted that there is a relation between these values and $G_{p 2 p}$. Comparing Figure 9 and Figure 11, it can be concluded that $G_{p 2 p}$ increases when correlation does.

3.3. Perspective. In this work, the channel features and a first approach of the TR technique have been studied in a high accurate IB2IB scenario. Future works should obtain results in this scenario in other experimental setups such as

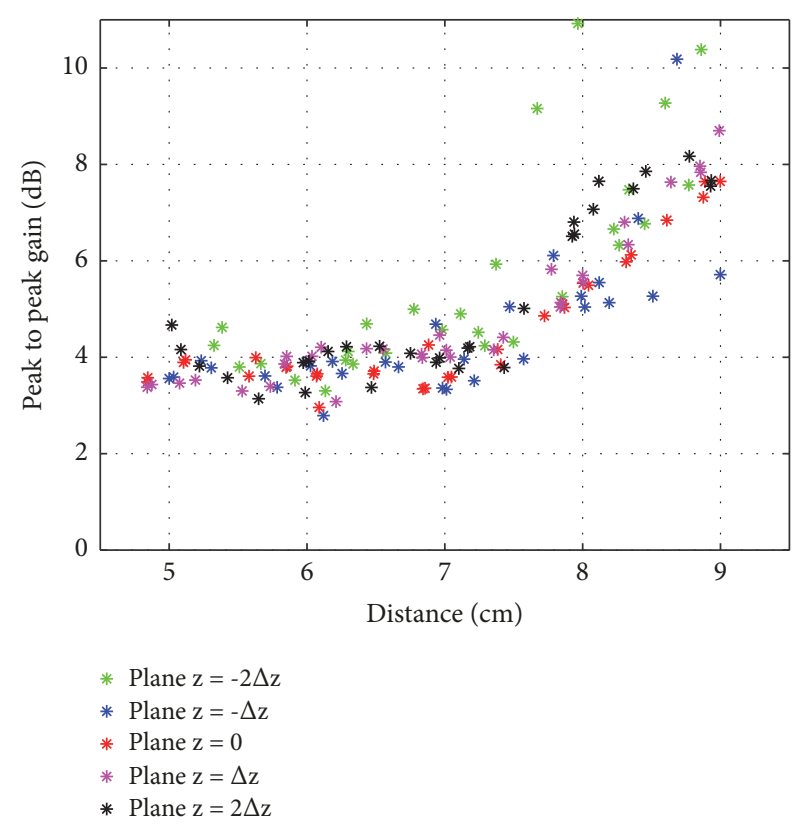

FIGURE 11: Peak to peak gain at different $\mathrm{Z}$ planes.

in vivo experiments by using animal subjects. In this way, the findings obtained in this work could be compared as well.

In order to obtain a standardized channel model for future in-body networks, more and more validations should be performed within the UWB frequency range. Therefore, the values obtained in this measurement campaign should be compared with those obtained in different parts of the body. On the other hand, the TR technique should be also tested in realistic implanted multisensor networks by using sophisticated transmission-reception environments as in [39].

\section{Conclusions}

In this paper, results obtained from a high accurate phantombased measurement setup are presented and discussed. This experimental setup reproduces the transmission between implanted nodes in the abdominal region. On the one hand, channel performance within UWB frequencies $(3.1-5.1 \mathrm{GHz})$ is assessed for the in-body to in-body (IB2IB) scenario. Our results evidenced that the path loss models could be well fitted by a linear approximation model regardless of the $\mathrm{Z}$ plane considered. Besides, the correlation in transmission and reception is evaluated. Uncorrelated channel impulse responses in transmission are achieved when the nodes are separated $4 \mathrm{~cm}$ at least. In reception, the receivers have to be more than $2 \lambda$ away to reach uncorrelation. On the other hand, the reciprocity of the channel has been studied and proved since the propagation medium is not the free space. Inasmuch as the channel is reciprocal, it allows the use of diversity techniques to compensate the high losses of the propagation medium. Using the Time Reversal processing technique, a gain between 4 and $11 \mathrm{~dB}$ can be achieved. This enhancement implies that the distance between sensors could increase from 
1 to $2 \mathrm{~cm}$, i.e., $11-22 \%$ from the case without applying Time Reversal processing. As expected, this gain is higher when the correlation in transmission decreases.

\section{Data Availability}

The data used to support the findings of this study are available from the corresponding author upon request.

\section{Conflicts of Interest}

The authors declare that there are no conflicts of interest regarding the publication of this paper.

\section{Acknowledgments}

This work was supported by the Programa de Ayudas de Investigación y Desarrollo (PAID-01-16) from Universitat Politècnica de València and by the Ministerio de Economía y Competitividad, Spain (TEC2014-60258-C2-1-R), by the European FEDER funds.

\section{References}

[1] G. Iddan, G. Meron, A. Glukhovsky, and P. Swain, "Wireless capsule endoscopy," Nature, vol. 405, no. 6785, pp. 417-418, 2000.

[2] Z. Fireman, E. Mahajna, E. Broide et al., "Diagnosing small bowel Crohn's disease with wireless capsule endoscopy," Gut, vol. 52, no. 3, pp. 390-392, 2003.

[3] H. Burri and D. Senouf, "Remote monitoring and followup of pacemakers and implantable cardioverter defibrillators," Europace, vol. 11, no. 6, pp. 701-709, 2009.

[4] IEEE Standard for Local and metropolitan area networks - Part 15.6: Wireless Body Area Networks, pp. 1-271, 2012.

[5] W. G. Scanlon, J. Brian Burns, and N. E. Evans, "Radiowave propagation from a tissue-implanted source at $418 \mathrm{MHz}$ and 916.5 MHz," IEEE Transactions on Biomedical Engineering, vol. 47 , no. 4, pp. 527-534, 2000.

[6] R. Chavez-Santiago, C. Garcia-Pardo, A. Fornes-Leal et al., "Experimental path loss models for in-body communications within 2.36-2.5 GHz," IEEE Journal of Biomedical and Health Informatics, vol. 19, no. 3, pp. 930-937, 2015.

[7] A. Khaleghi, R. Chávez-Santiago, and I. Balasingham, "Ultrawideband pulse-based data communications for medical implants," IET Communications, vol. 4, no. 15, pp. 1889-1897, 2010.

[8] J. Wang and Q. Wang, "Channel Modeling and BER Performance of an implant UWB body area link," in Proceedings of the 2nd International Symposium on Applied Sciences in Biomedical and Communication Technologies, pp. 1-4, Bratislava, Slovakia, November 2009.

[9] S. Stoa, R. Chavez-Santiago, and I. Balasingham, "An ultra wideband communication channel model for capsule endoscopy," in Proceedings of the 2010 3rd International Symposium on Applied Sciences in Biomedical and Communication Technologies, ISABEL 2010, Rome, Italy, November 2010.

[10] C. Andreu, C. Garcia-Pardo, A. Fomes-Leal, M. Cabedo-Fabres, and N. Cardona, "UWB in-body channel performance by using a direct antenna designing procedure," in Proceedings of the 11th European Conference on Antennas and Propagation, EUCAP 2017, pp. 278-282, Paris, France, March 2017.

[11] A. Khaleghi, R. Chàvez-Santiago, and I. Balasingham, "Ultrawideband statistical propagation channel model for implant sensors in the human chest," IET Microwaves, Antennas \& Propagation, vol. 5, no. 15, p. 1805, 2011.

[12] D. Kurup, M. Scarpello, G. Vermeeren et al., "In-body path loss models for implants in heterogeneous human tissues using implantable slot dipole conformal flexible antennas," EURASIP Journal on Wireless Communications and Networking, vol. 2011, no. 1, p. 51, 2011.

[13] P. A. Floor, R. Chavez-Santiago, S. Brovoll et al., "In-Body to on-body ultrawideband propagation model derived from measurements in living animals," IEEE Journal of Biomedical and Health Informatics, vol. 19, no. 3, pp. 938-948, 2015.

[14] Y. Shimizu, D. Anzai, R. Chavez-Santiago, P. A. Floor, I. Balasingham, and J. Wang, "Performance Evaluation of an UltraWideband Transmit Diversity in a Living Animal Experiment," IEEE Transactions on Microwave Theory and Techniques, vol. 65, no. 7, pp. 2596-2606, 2017.

[15] D. Anzai, K. Katsu, R. Chávez-Santiago et al., "Experimental evaluation of implant UWB-IR transmission with living animal for body area networks," IEEE Transactions on Microwave Theory and Techniques, vol. 62, no. 1, pp. 183-192, 2014.

[16] R. Chavez-Santiago, C. Garcia-Pardo, A. Fornes-Leal, A. VallesLluch, I. Balasingham, and N. Cardona, "Ultra wideband propagation for future in-body sensor networks," in Proceedings of the 2014 25th IEEE Annual International Symposium on Personal, Indoor, and Mobile Radio Communication, IEEE PIMRC 2014, pp. 2160-2163, Washington, DC, USA, September 2014.

[17] C. Chou, G. Chen, A. W. Guy, and K. H. Luk, "Formulas for preparing phantom muscle tissue at various radiofrequencies," Bioelectromagnetics, vol. 5, no. 4, pp. 435-441, 1984.

[18] A. Y. Cheung, "Experimental Development of Simulated Biomaterials for Dosimetry Studies of Hazardous Microwave Radiation," IEEE Transactions on Microwave Theory and Techniques, vol. 24, no. 10, pp. 669-673, 1976.

[19] H. Yamamoto, J. Zhou, and T. Kobayashi, "Ultra wideband electromagnetic phantoms for antennas and propagation studies," IEICE Transactions on Fundamentals of Electronics, Communications and Computer Sciences, vol. E91-A, no. 11, pp. 3173-3182, 2008.

[20] M. Lazebnik, E. L. Madsen, G. R. Frank, and S. C. Hagness, "Tissue-mimicking phantom materials for narrowband and ultrawideband microwave applications," Physics in Medicine and Biology, vol. 50, no. 18, pp. 4245-4258, 2005.

[21] T. Yilmaz, R. Foster, and Y. Hao, "Broadband tissue mimicking phantoms and a patch resonator for evaluating noninvasive monitoring of blood glucose levels," IEEE Transactions on Antennas and Propagation, vol. 62, no. 6, pp. 3064-3075, 2014.

[22] C. Gabriel, "Compilation of the Dielectric Properties of Body Tissues at RF and Microwave Frequencies," Tech. Rep., Brooks Air Force Base, San Antonio, TX, USA, 1996.

[23] S. Gezici, Z. Tian, G. B. Giannakis et al., "Localization via ultrawideband radios: A look at positioning aspects of future sensor networks," IEEE Signal Processing Magazine, vol. 22, no. 4, pp. 70-84, 2005.

[24] Q. Wang, R. Hahnel, and D. Plettemeier, "Diversity reception evaluation for in-body to on-body communication channel in UWB low band," in Proceedings of the 8th International Conference on Body Area Networks, pp. 270-276, October 2013. 
[25] M. Marinova, A. Thielens, E. Tanghe et al., "Diversity performance of off-body MB-OFDM UWB-MIMO," Institute of Electrical and Electronics Engineers. Transactions on Antennas and Propagation, vol. 63, no. 7, pp. 3187-3197, 2015.

[26] J. Shi, D. Anzai, and J. Wang, "Channel modeling and performance analysis of diversity reception for implant UWB wireless link," IEICE Transactions on Communications, vol. E95-B, no. 10, pp. 3197-3205, 2012.

[27] P. Pajusco and P. Pagani, "On the use of uniform circular arrays for characterizing UWB time reversal," IEEE Transactions on Antennas and Propagation, vol. 57, no. 1, pp. 102-109, 2009.

[28] N. Cardona Marcet, S. Castelló Palacios, A. Fornés Leal, C. García Pardo, and A. Vallés Lluch, "Synthetic Model of Biological Tissues for Evaluating the Wireless Transmission of Electromagnetic Waves," Spanish Patent PCT/ES2016/070912, Jun-2017.

[29] R. Chavez-Santiago, K. Sayrafian-Pour, A. Khaleghi et al., "Propagation models for IEEE 802.15.6 standardization of implant communication in body area networks," IEEE Communications Magazine, vol. 51, no. 8, pp. 80-87, 2013.

[30] P. S. Hall and Y. Hao, "Antennas and propagation for bodycentric wireless communications," p. 258, 2006.

[31] Q. Wang, K. Wolf, and D. Plettemeier, "An UWB capsule endoscope antenna design for biomedical communications," in Proceedings of the 2010 3rd International Symposium on Applied Sciences in Biomedical and Communication Technologies, ISABEL 2010, pp. 1-6, Rome, Italy, November 2010.

[32] K. Y. Yazdandoost and R. Miura, "Miniaturized UWB implantable Antenna for Brain-Machine-Interface," in Proceedings of the 9th European Conference on Antennas and Propagation, EuCAP 2015, pp. 1-5, May 2015.

[33] C. Andreu, S. Castello-Palacios, C. Garcia-Pardo, A. FornesLeal, A. Valles-Lluch, and N. Cardona, "Spatial In-Body Channel Characterization Using an Accurate UWB Phantom," IEEE Transactions on Microwave Theory and Techniques, vol. 64, no. 11, pp. 3995-4002, 2016.

[34] R. K. Makoto Kawasaki, "A TOA Based Positioning Technique of Medical Implanted Devices," in Proceedings of the 3rd International Symposium on Medical Information \& communication Technology (ISMICT), 2008.

[35] T. S. Rappaport, Wireless Communications: Principles and Practice, Dorling Kindersley, 2009.

[36] K. Pahlavan and A. H. Levesque, Wireless Information Networks, John Wiley \& Sons, 2005.

[37] R. C. Qiu, C. Zhou, N. Guo, and J. Q. Zhang, “Time reversal with MISO for ultrawideband communications: experimental results," IEEE Antennas and Wireless Propagation Letters, vol. 5, no. 1, pp. 269-273, 2006.

[38] C. A. Balanis, Antenna Theory: Analysis and Design, John Wiley \& Sons, 3rd edition, 2005.

[39] H. Ando, K. Takizawa, T. Yoshida, K. Matsushita, M. Hirata, and T. Suzuki, "Wireless Multichannel Neural Recording with a 128-Mbps UWB Transmitter for an Implantable Brain-Machine Interfaces," IEEE Transactions on Biomedical Circuits and Systems, vol. 10, no. 6, pp. 1068-1078, 2016. 


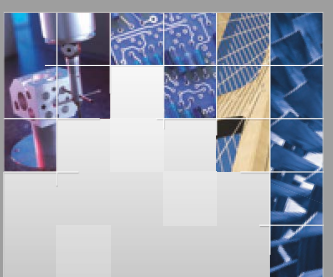

\section{Enfincering}
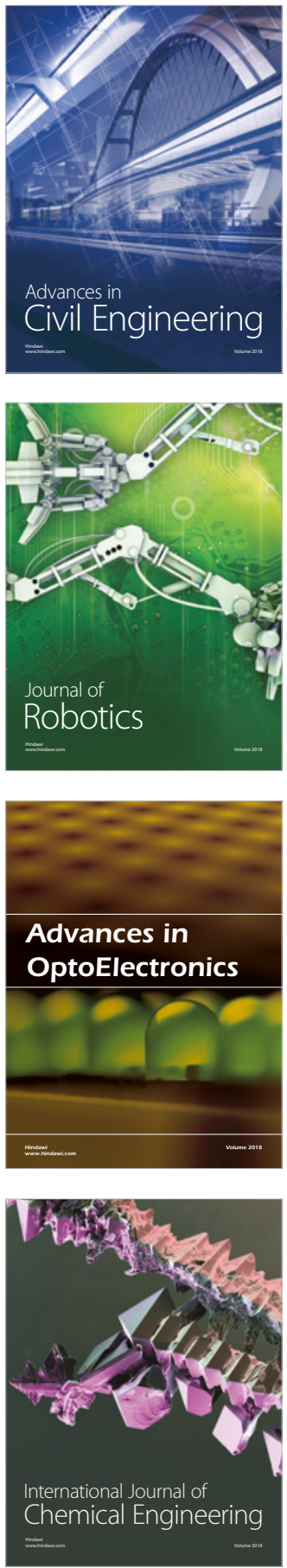

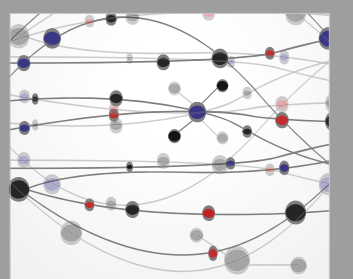

\section{Rotating \\ Machinery}

The Scientific World Journal

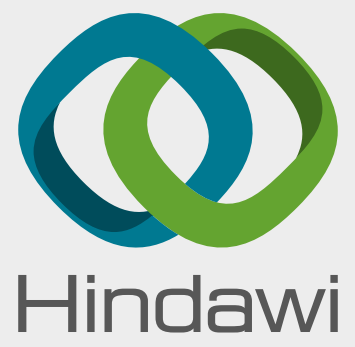

Submit your manuscripts at

www.hindawi.com
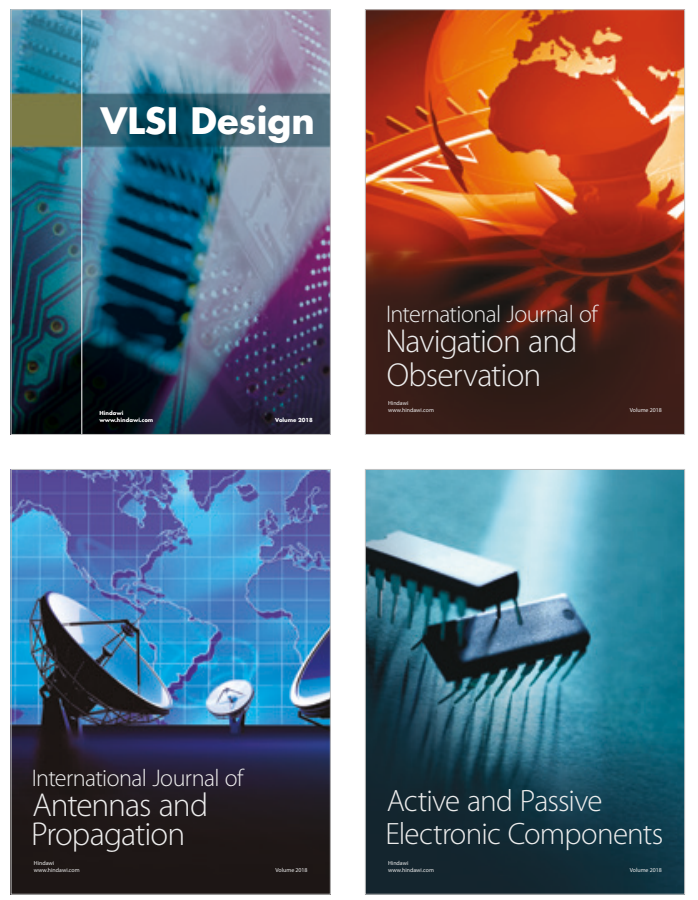
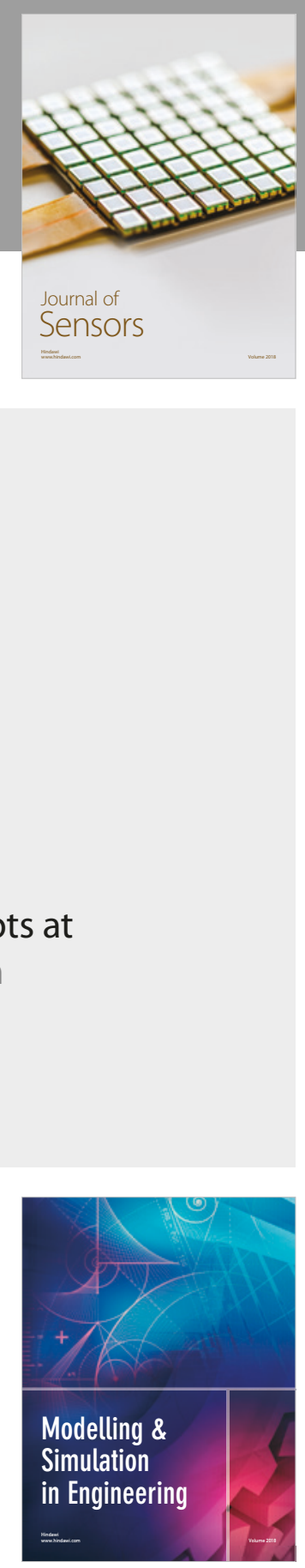

\section{Advances \\ Multimedia}
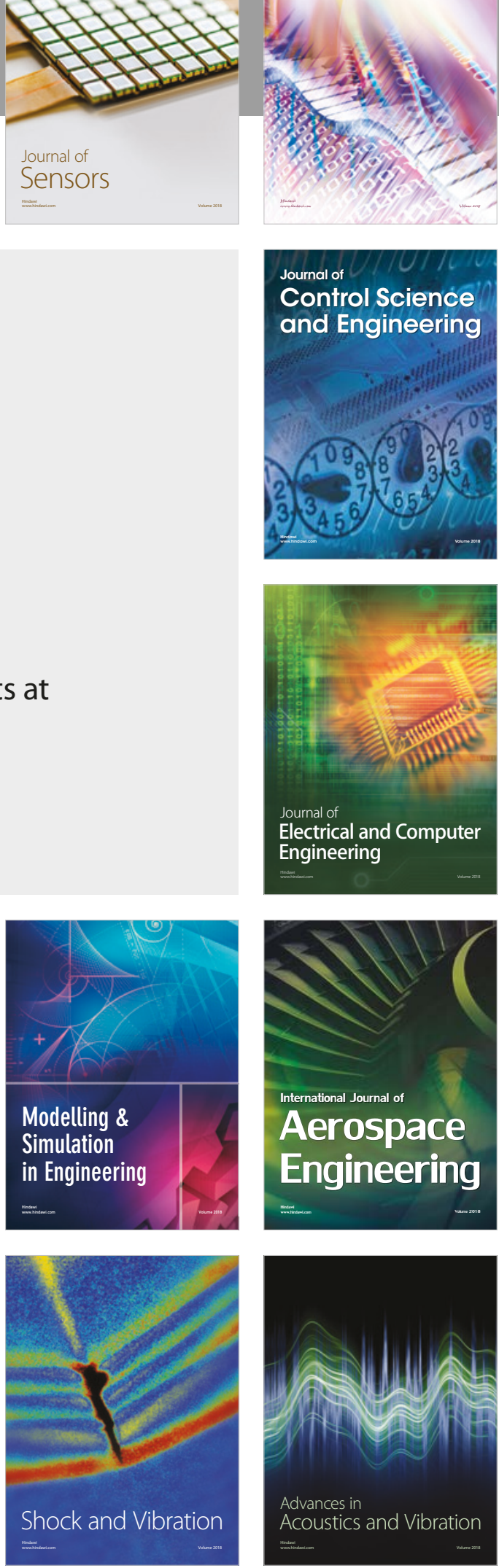